Case

Report

\title{
Uterine Intravenous Leiomyomatosis with Right Ventricular Extension
}

\author{
Masaya Sogabe, MD, Koji Kawahito, MD, Kei Aizawa, MD, \\ Hirotaka Sato, MD, and Yoshio Misawa, MD
}

\begin{abstract}
Intravenous leiomyomatosis is a rare neoplastic condition characterized by the benign intravascular proliferation of smooth muscle cells originating from either the uterine venous wall or a uterine leiomyoma. In the present report, we describe the case of a 45-year-old woman without a history of gynaecological surgeries, who was referred to our institution due to repeated syncopal attacks. Computed tomography indicated the presence of an intravenous leiomyoma originating from the uterus and extending to the inferior vena cava, right atrium, and right ventricle. The patient was successfully treated by cardiotomy, which was performed under hypothermic circulatory arrest, and laparotomy in a single-stage operation. She continued to recover and did not exhibit any recurrence at the 10-month follow-up.
\end{abstract}

Keywords: intravenous leiomyomatosis, uterus, cardiac extension

\section{Introduction}

Although uterine leiomyomatosis is a common condition among women, the occurrence of intravenous leiomyomatosis (IVL) with intracardiac extension is rare. ${ }^{1,2)}$ As intracardiac leiomyomatosis may result in cardiac symptoms and may cause sudden death, prompt and adequate surgical intervention is mandatory. In the present report, we describe a case of uterine intravenous leiomyomatosis with cardiac extension that was successfully treated with a single-stage operation.

\section{Case Report}

A 45-year-old woman was admitted to the hospital with a 3-month history of repeated syncopal attacks.

Department of Cardiovascular Surgery, Jichi Medical University, Shimotsuke, Tochigi, Japan

Received: October 30, 2013; Accepted: November 12, 2013 Corresponding author: Koji Kawahito, MD. Department of Cardiovascular Surgery, Jichi Medical University, 3311-1 Yakushiji, Shimotsuke, Tochigi 329-0498, Japan

Email:kj_kawahito@msn.com

(C)2014 The Editorial Committee of Annals of Thoracic and Cardiovascular Surgery. All rights reserved.
She had no history of gynaecological surgeries. On physical examination, a large abdominal mass was palpable in the lower abdomen and a systolic murmur was noted in Erb's area on auscultation. On pelvic computerised tomography (CT), a heterogeneous pelvic mass, approximately $13 \times 9 \times 8 \mathrm{~cm}$ in size, that suggested the presence of a uterine leiomyoma was noted (Fig. 1A). CT of the chest and abdomen indicated the presence of a hypodense mass emerging from the uterine tumour that extended to the inferior vena cava via the right ovarian vein, and then extended into the right heart (Fig. 1A and 1B). In addition, echocardiography indicated a mass floating within the inferior vena cava, extending into the right atrium. Furthermore, transthoracic echocardiography showed a mobile cord-like mass extending from the inferior vena cava to the right atrium that moved back and forth through the tricuspid orifice into the right ventricle (Fig. 2).

Surgery was performed through a median sternotomy and midline laparotomy, with the patient under cardiopulmonary bypass with deep hypothermia and circulatory arrest. First, the abdomen was incised. The uterus was found to be enlarged with an intramural leiomyoma. Moreover, the tumour was noted to have spread to the inferior vena cava through the right ovarian vein. We 


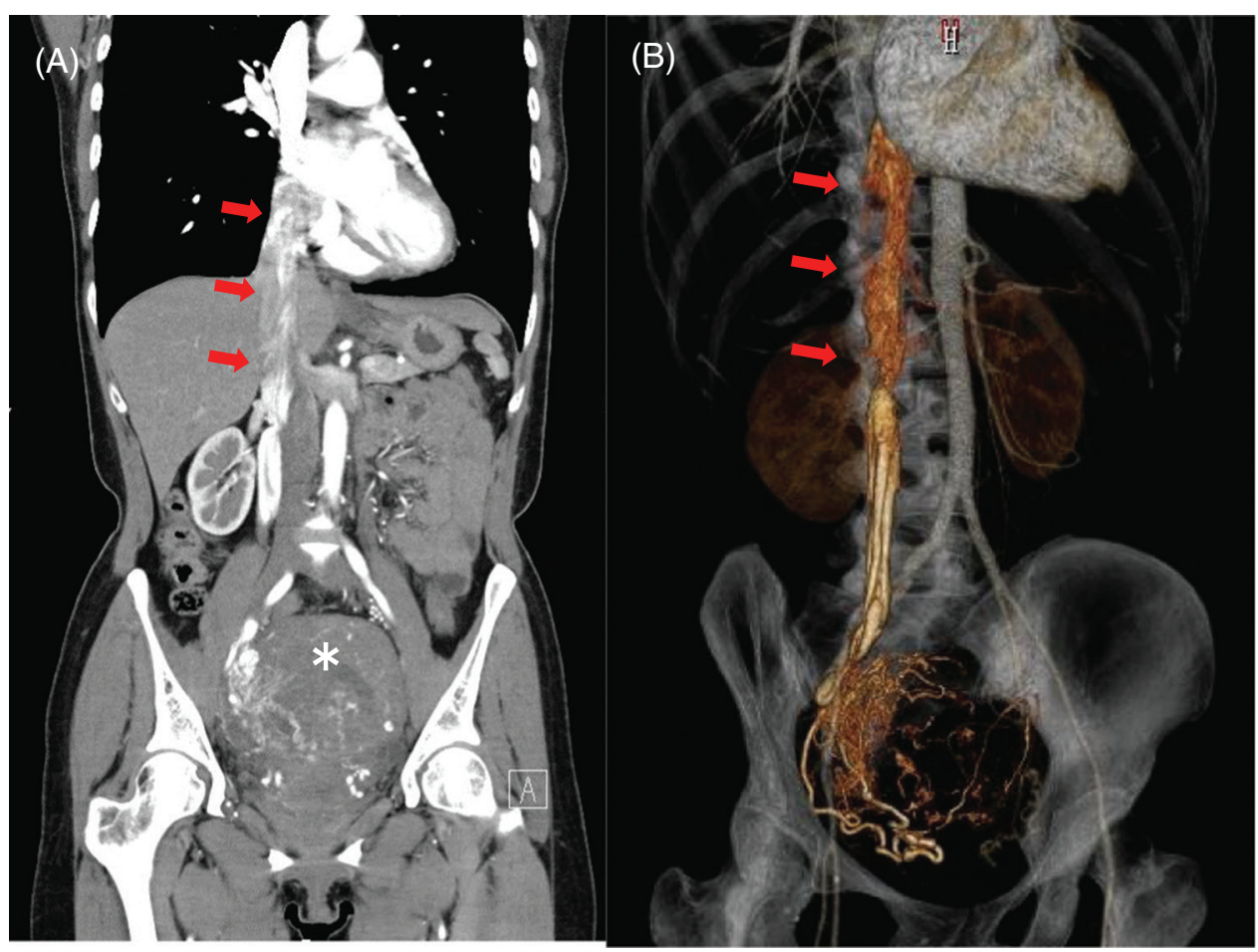

Fig. 1 A computed tomography image showing a large heterogeneous pelvic mass (A) (asterisk) and a hypodense mass emerging from the right ovarian vein, extending to the inferior vena cava, and then extending into the right heart (arrows) (A, B).
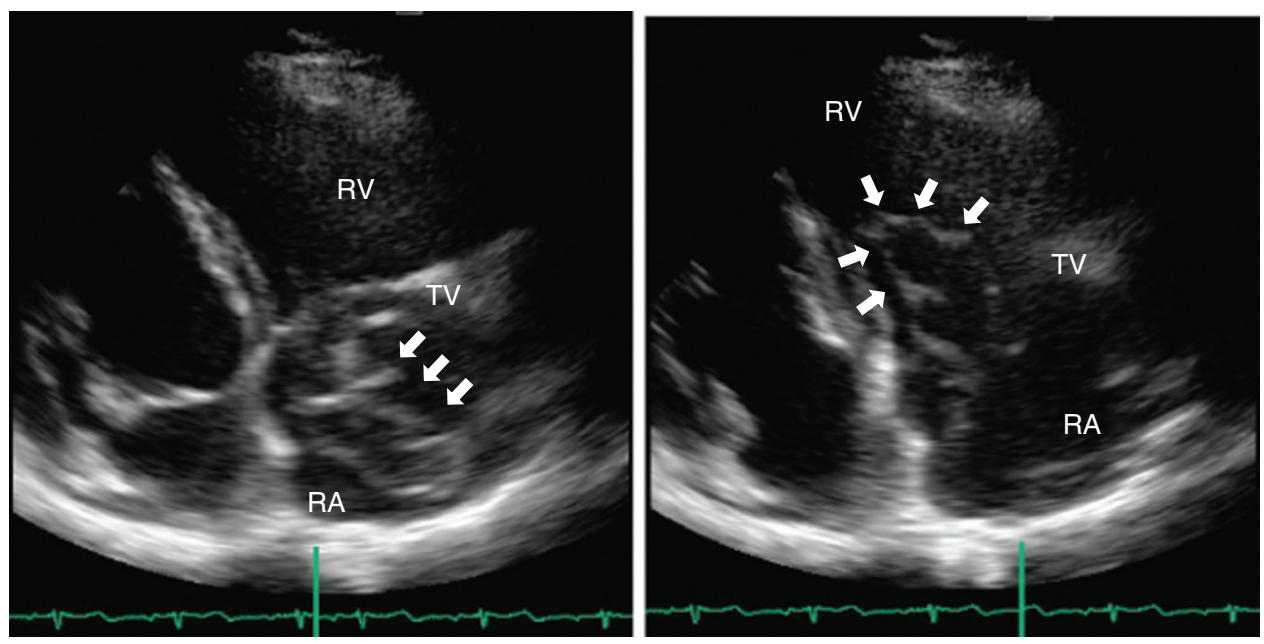

Fig. 2 Transthoracic echocardiography indicated a mobile cord-like mass extending from the inferior vena cava to the right atrium (RA) and protruding through the tricuspid valve (TV) into the right ventricle (RV) (arrows).

divided the tumour into two portions via a surgical procedure at the site where it penetrated into the right ovarian vein. Subsequently, total abdominal hysterectomy with bilateral salpingo-oophorectomy, including the removal of the huge tumour mass, was performed. Thereafter, the infraphrenic vena cava was isolated.

A median sternotomy was then performed and cardiopulmonary bypass was established by cannulating the ascending aorta with venous drainage through the right femoral vein and the superior vena cava. The right atrium was incised with the patient under deep hypothermic circulatory arrest. The intracardiac mass was found to be floating freely without involvement of the cardiac structure (Fig. 3A). Immediately thereafter, the right ovarian vein was cut-off and the inferior vena cava was incised via a venotomy. The intracardiac and intracaval component 


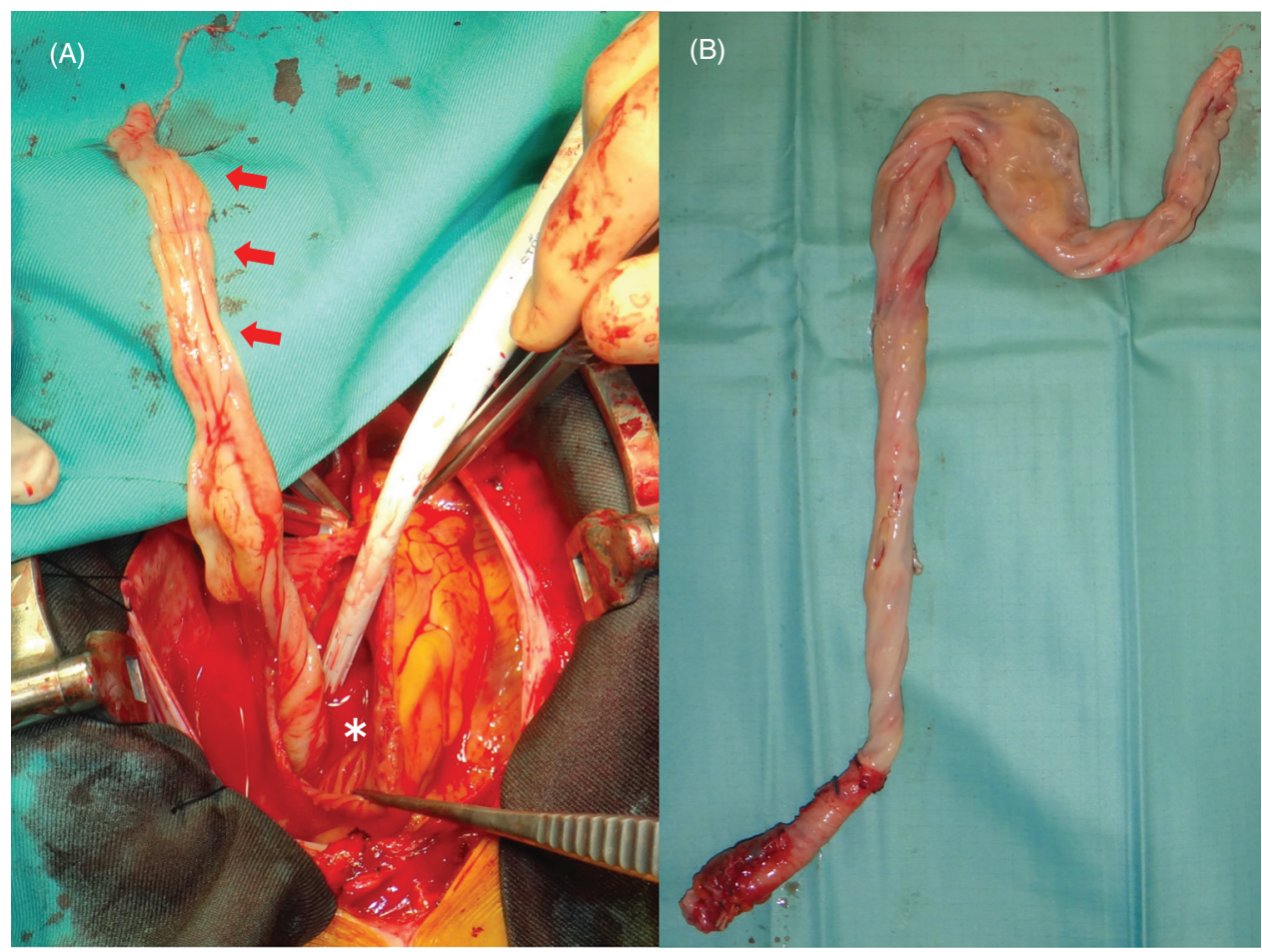

Fig. 3 Intraoperative image showing the intracardiac components of the tumour (arrows) (A). Asterisk indicates the location of the right atriotomy. The operative specimen-a 30-cm-long, grey-white stromal tumour mass — exhibiting intracardiac and intracaval components (B).

of the tumour were drawn out downward through the venotomy incision. During this procedure, no adherence of the tumor was noted. After closure of the venotomy and right atriotomy incisions by using sutures, rewarming was initiated from a minimum temperature of $23^{\circ} \mathrm{C}$. The total cardiopulmonary bypass time was $120 \mathrm{~min}$, aortic cross clamp time was $35 \mathrm{~min}$, and circulatory arrest time was $10 \mathrm{~min}$.

On examination, the tumour was found to be firm and rubbery, without any tumour thrombi or areas of friable tissue. The operative specimen with intracardiac and intracaval components was a $30-\mathrm{cm}$ long, grey-white, stromal tumour mass (Fig. 3B). Histologic examination indicated smooth muscle cell proliferation consistent with a diagnosis of intravenous leiomyomatosis. The patient's postoperative course was uneventful, and she was discharged on the 10th postoperative day. She continued to recover well and did not exhibit any recurrence at the 10-month follow-up.

\section{Discussion}

IVL is a rare neoplastic disease, which is characterized by intravenous growth of histologically benign, smooth muscle tumours arising from either a uterine myoma or from the wall of a uterine vessel.,3) With regard to the aetiology of IVLs, two main theories have been proposed. The first theory indicates that the tumour originates directly from the vein walls, whereas the second suggests that a uterine leiomyoma, as the primary tumour, causes intravascular projections into an adjacent venous channel. ${ }^{5,6)}$ In the present case, we noted that the IVL originated from the main uterine leiomyoma and extended to the vascular lumen, which may support the second theory. With regard to the extension pathway, the tumour is usually known to enter through the lumen of the iliac vein, and grow into the inferior vena cava. ${ }^{7)}$ However, in certain cases, the ovarian vein serves as an alternative route to the inferior vena cava, as in the present case.

IVL is histologically benign and characterised by slow growth. Even with intravenous tumour extension as far as the inferior vena cava, symptoms may be absent until cardiac insufficiency developed due to intracardiac extension. Although the clinical presentation depends on the extension and size of the tumour, right ventricular extension may cause complete outflow obstruction. ${ }^{8)}$ In the present case, the patient had a history of repeated syncopal attacks probably due to the obstructive effect of 
the tumour on the tricuspid orifice. In such cases, the patient should undergo prompt surgical resection due to the risk of sudden death.

The operative approach requires careful consideration with the aim of complete resection of neoplastic tissue, because recurrence appears to be rare when complete resection is achieved, while incomplete removal leads to recurrence in one-third of patients. ${ }^{1)}$ Although choosing between one- or two-stage treatments in such cases is controversial, ${ }^{1,2)}$ a decision should be made while considering the patient's clinical condition. One-stage surgery enables complete surgical resection without any residual tumour; however, it places a much heavier burden on the patient. If the patient is ineligible for single-stage surgery because of a poor clinical condition due to cardiac and pulmonary comorbidities, a two-stage operation may be optimal. In the present case, the patient's clinical condition was relatively good, and therefore, a radical one-stage operation was performed and a positive outcome was obtained.

\section{Conclusion}

We performed one-stage tumor extraction in a patient with intravenous leiomyomatosis with cardiac extension. The postoperative cause was uneventful and did not exhibit any recurrence at the 10-month follow-up.

\section{Disclosure Statement}

None.

\section{References}

1) Clay TD, Dimitriou J, McNally OM, et al. Intravenous leiomyomatosis with intracardiac extension-a review of diagnosis and management with an illustrative case. Surg Oncol 2013; 22: e44-52.

2) Li B, Chen X, Chu YD, et al. Intracardiac leiomyomatosis: a comprehensive analysis of 194 cases. Interact Cardiovasc Thorac Surg 2013; 17: 132-8.

3) Kullo IJ, Oh JK, Keeney GL, et al. Intracardiac leiomyomatosis: echocardiographic features. Chest 1999; 115: 587-91.

4) Yu L, Shi E, Gu T, et al. Intravenous leiomyomatosis with intracardiac extension: a report of two cases. J Card Surg 2011; 26: 56-60.

5) Norris HJ, Parmley T. Mesenchymal tumors of the uterus. V. Intravenous leiomyomatosis. A clinical and pathologic study of 14 cases. Cancer 1975; 36: 2164-78.

6) Borland DS, Wotring JW. Intravenous leiomyomatosis of the uterus and broad ligament. Report of a case. Am J Clin Pathol 1964; 42: 182-8.

7) Lam PM, Lo KW, Yu MY, et al. Intravenous leiomyomatosis: two cases with different routes of tumor extension. J Vasc Surg 2004; 39: 465-9.

8) Roman DA, Mirchandani H. Intravenous leiomyoma with intracardiac extension causing sudden death. Arch Pathol Lab Med 1987; 111: 1176-8. 